\title{
nature
}

nanotechnology

\section{Future challenges for Asia}

\author{
The scientific output of the Asia-Pacific region is growing, especially in nanoscience and \\ technology, but there is scope for greater collaboration and networking between researchers.
}

Getting researchers from different countries and different disciplines to work together can be difficult. Different languages are spoken - both literally and scientifically; different funding agencies are involved; different journals are preferred; and then there are centuries of history and squabbles about intellectual property to sort out. In Europe, the European Union is now on its seventh framework programme for research and technological development ${ }^{1}$ - with collaboration between different member states being a pre-condition for many parts of the programme - and researchers still complain about it.

So if cross-border collaboration is difficult on a continent where an intergovernmental organization has existed since 1957, imagine how hard it must be in the Asia-Pacific region, where there is no comparable multinational body, where the would-be member states are much further apart (especially when India, Australia and New Zealand are included), and where the differences in population and scientific infrastructure between the two ends of the scale vastly exceed those found in Europe. It is little wonder that the level of networking and collaboration in the region remains relatively modest, which is why Nature Publishing Group organized a conference ${ }^{2}$ on this topic in Tokyo last month. One of the reasons for the low level of networking is that many researchers in Asia-Pacific prefer to collaborate with laboratories in the US and Europe, rather than institutions closer to home. As Edison Liu of the Genome Institute of Singapore put it: "A collaboration with Boston is more likely to get you noticed by your chair" (ref. 3).

The conference also heard that the scientific output of the Asia-Pacific region is increasing, particularly in the physical sciences, with China providing most of the growth, and in nanoscience and technology it is estimated that almost half of all papers now come from Asia-Pacific. It is true that the quantity of papers from China is growing faster than their quality, but more Chinese papers are appearing in leading journals.

Nanotechnology was one of four topics discussed in detail at the conference - the others being stem cells, molecular biology and biotechnology, and web technologies and infrastructures for networking - and it was certainly the smallest, not just in the literal sense: unlike 'big physics' and 'big biology', there are no mega-author lists or enormous data sets in nanotechnology. Moreover, many of the most interesting problems in nanoscience and technology require researchers from very different backgrounds to work together.

\section{Many researchers in Asia-} Pacific prefer to collaborate with laboratories in the US and Europe, rather than institutions closer to home.

However, nanotechnology does resemble big physics in the sense that it sometimes requires expensive instruments for fabrication and characterization that are beyond the reach of many groups, and there was widespread agreement that it is essential to make the best use of such equipment by operating it at full capacity and making it available to as many researchers as possible.

In general, 'keep it simple' was the main message to emerge on the subject of networking and collaboration. At the institutional level this was illustrated by the flat and flexible structures described by Jackie Ying of the Institute of Bioengineering and Nanotechnology in Singapore, where a project-based approach naturally brings together researchers with different backgrounds. For collaborations - either between laboratories or between countries - a bilateral or trilateral approach appears to work best. There was also a general consensus that in international collaborations, funds and resources have to flow from rich nations to not-so-rich ones.

Several delegates felt that there are too many national meetings and that there is a need for a smaller number of larger meetings that cover the whole Asia-Pacific region. Speakers and delegates also emphasized the need for further work on standards and metrology, and the need for more research on the environmental, health and safety aspects of nanomaterials. Although this has long been seen as a priority for the nanotechnology community for many reasons ${ }^{4}$ - such as gaining public acceptance and generating investment - progress has been much slower than necessary and, moreover, the possibility that different regulations in different countries could hinder international collaboration has not been widely recognized.

Although nanoscience and technology do not demand the level of international collaboration that is de rigeur in particle physics, genetics and climate science, there are global issues and challenges on which the nanotechnology community can focus and make an impact. Joining forces to work on new energy sources and environmental technology $y^{5}$ for the Asia-Pacific region in general - and China and India in particular - would be a good place to start.

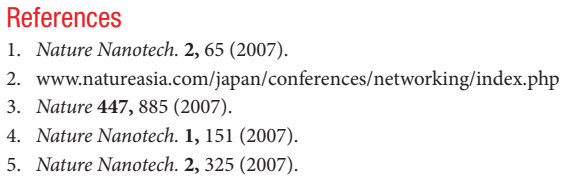

